\title{
MODEL FOR IDENTIFYING VOLATILITY IN EQUITY MARKET WITH THE HELP OF NSE LISTED STOCKS WITH SPECIAL REFERENCE TO APOLLO TYRES, ASIAN PAINTS AND ASHOK LEYLAND DURING POST COVID-19 IN INDIA- A CASE STUDY
}

\author{
R Amuthan \\ Associate Professor, Department of Management Studies, Karunya (Deemed) University, \\ Coimbatore, Tamilnadu, India. \\ V. Lawrance \\ Professor, Department of Management Studies, Karunya (Deemed) University, \\ Coimbatore, Tamilnadu, India.
}

\begin{abstract}
This study covers the whole gamut of volatility in terms of Share price increase or decrease. The centre of coverage over here in this study is COVID-19. We all have to strongly admit that COVID has created much more crucified impacts on all businesses including stock market trading. 100 Trading Days Cash Market data and 90 trading days Future Price data were taken in to account. Volatility is being computed with the help of Excel application and it is found that those stocks experienced low upswings are said to be high risky stocks and these stocks are not fit enough to buy. These share prices are further backed up by Cash flows, Book Value Per share, Earnings Per share. These financial variables' quarterly growth history from the start of Lock down is checked out and if the growth is negative, the concerned stock is classified as risk and it is not fit to buy.
\end{abstract}

Key words: Volatility, upswing, downswing, Fit to Buy for Investment and Trading Cite this Article: R Amuthan and V. Lawrance, Model for Identifying Volatility in Equity Market with the Help of NSE Listed Stocks with Special Reference to Apollo Tyres, Asian Paints and Ashok Leyland during Post Covid-19 in India - A Case Study, International Journal of Management, 11(12), 2020, pp 1630-1641.

http://iaeme.com/Home/issue/IJM?Volume=11\&Issue $=12$ 
Model for Identifying Volatility in Equity Market with the Help of NSE Listed Stocks with Special Reference to Apollo Tyres, Asian Paints and Ashok Leyland during Post Covid-19 in India - A Case

\section{INTRODUCTION}

Study

It is a rate at which the price of a security increases or decreases for a given set of returns. Volatility is measured by calculating the standard deviation of the annualized returns over a given period of time. It shows the range to which the price of a security may increase or decrease. Volatility measures the risk of a security. It is used in option pricing formula to gauge the fluctuations in the returns of the underlying assets. Volatility indicates the pricing behavior of the security and helps estimate the fluctuations that may happen in a short period of time. If the prices of a security fluctuate rapidly in a short time span, it is termed to have high volatility. If the prices of a security fluctuate slowly in a longer time span, it is termed to have low volatility.

\subsection{Statement of Problem}

In Equity Market, detecting the real stocks which are yielding returns is found to be difficult on account of very high volatility in the Market. Hence there is the strong and reliable analysis are required based on certain criteria.

\subsection{Objectives of the Study}

To describe the model to find out Highly Volatile and Less Volatile Stocks

\subsection{Need for the Study}

- To inform novice investors about the whole landscape of stock market fluctuations

- To tell them how to make money out of stock market investments

\subsection{Scope of Study}

This study explains the methodology of finding stock market volatility with the evidence of Apollo Tyres Limited, Asian Paints Limited and Ashok Leyland Limited. 101 trading days in Cash Market and 90 Trading Days in Derivative Market were taken to prove the volatility impacts.

\subsection{Limitations}

This study derives its findings and conclusions only by means of analyzing Financial Data. All drawbacks using financial data will be reflected in this study also.

\section{ANALYSIS AND INTERPETATION}

\subsection{Apollo Tyres}

Apollo Tyres Ltd, with its corporate headquarters in Gurgaon, India, is in the business of manufacture and sale of Tyres since its inception in 1972. Over the years, the company has grown manifold, establishing its footprint across the globe. The company has manufacturing presence in Asia and Europe with 8 modern tyre facilities and exports to over 118 countries. Powered by its key brands - Apollo and Vredestein, the company offers a comprehensive product portfolio spread across passenger car, light truck, truck-bus, off highway and bicycle tyres, retreading material and retreaded tyres. 
Table 1 Closing Price on Cash Market

\begin{tabular}{|c|c|c|c|}
\hline Date & Closing Price & change in closing price & Swings \\
\hline $14-09-2020$ & 125.55 & & \\
\hline $11 / 9 / 2020$ & 116.2 & -9.35 & Down Swing \\
\hline $10 / 9 / 2020$ & 116.25 & 0.05 & Up Swing \\
\hline $9 / 9 / 2020$ & 113.4 & -2.85 & Down Swing \\
\hline $8 / 9 / 2020$ & 113.3 & -0.1 & Down Swing \\
\hline $7 / 9 / 2020$ & 118.8 & 5.5 & Up Swing \\
\hline $4 / 9 / 2020$ & 120.95 & 2.15 & Up Swing \\
\hline $3 / 9 / 2020$ & 123.7 & 2.75 & Up Swing \\
\hline $2 / 9 / 2020$ & 122.55 & -1.15 & Down Swing \\
\hline $1 / 9 / 2020$ & 122.45 & -0.1 & Down Swing \\
\hline $31-08-2020$ & 123.55 & 1.1 & Up Swing \\
\hline $28-08-2020$ & 133.55 & 10 & Up Swing \\
\hline $27-08-2020$ & 135.5 & 1.95 & Up Swing \\
\hline $26-08-2020$ & 132 & -3.5 & Down Swing \\
\hline $25-08-2020$ & 126.85 & -5.15 & Down Swing \\
\hline $24-08-2020$ & 128.45 & 1.6 & Up Swing \\
\hline $21-08-2020$ & 131.25 & 2.8 & Up Swing \\
\hline $20-08-2020$ & 128.75 & -2.5 & Down Swing \\
\hline $19-08-2020$ & 127.9 & -0.85 & Down Swing \\
\hline $18-08-2020$ & 128.45 & 0.55 & Up Swing \\
\hline $17-08-2020$ & 129.35 & 0.9 & Up Swing \\
\hline $14-08-2020$ & 129.95 & 0.6 & Up Swing \\
\hline $13-08-2020$ & 131.85 & 1.9 & Up Swing \\
\hline $12 / 8 / 2020$ & 129.4 & -2.45 & Down Swing \\
\hline $11 / 8 / 2020$ & 126.05 & -3.35 & Down Swing \\
\hline $10 / 8 / 2020$ & 127.4 & 1.35 & Up Swing \\
\hline $7 / 8 / 2020$ & 123.55 & -3.85 & Down Swing \\
\hline $6 / 8 / 2020$ & 115.5 & -8.05 & Down Swing \\
\hline $5 / 8 / 2020$ & 115.9 & 0.4 & Up Swing \\
\hline $4 / 8 / 2020$ & 115.15 & -0.75 & Down Swing \\
\hline $3 / 8 / 2020$ & 108.1 & -7.05 & Down Swing \\
\hline $31-07-2020$ & 108.25 & 0.15 & Up Swing \\
\hline $30-07-2020$ & 108.3 & 0.05 & Up Swing \\
\hline $29-07-2020$ & 110.35 & 2.05 & Up Swing \\
\hline $28-07-2020$ & 110.75 & 0.4 & Up Swing \\
\hline $27-07-2020$ & 106.7 & -4.05 & Down Swing \\
\hline $24-07-2020$ & 109 & 2.3 & Up Swing \\
\hline $23-07-2020$ & 109.45 & 0.45 & Up Swing \\
\hline $22-07-2020$ & 109.65 & 0.2 & Up Swing \\
\hline $21-07-2020$ & 110.05 & 0.4 & Up Swing \\
\hline $20-07-2020$ & 109.75 & -0.3 & Down Swing \\
\hline $17-07-2020$ & 109.5 & -0.25 & Down Swing \\
\hline $16-07-2020$ & 109.45 & -0.05 & Down Swing \\
\hline $15-07-2020$ & 112.15 & 2.7 & Up Swing \\
\hline $14-07-2020$ & 110.45 & -1.7 & Down Swing \\
\hline $13-07-2020$ & 114.1 & 3.65 & Up Swing \\
\hline $10 / 7 / 2020$ & 113.2 & -0.9 & Down Swing \\
\hline $9 / 7 / 2020$ & 115.4 & 2.2 & Up Swing \\
\hline $8 / 7 / 2020$ & 116 & 0.6 & Up Swing \\
\hline $7 / 7 / 2020$ & 117.3 & 1.3 & Up Swing \\
\hline $6 / 7 / 2020$ & 117.8 & 0.5 & Up Swing \\
\hline $3 / 7 / 2020$ & 114.25 & -3.55 & Down Swing \\
\hline $2 / 7 / 2020$ & 110.85 & -3.4 & Down Swing \\
\hline $1 / 7 / 2020$ & 109.75 & -1.1 & Down Swing \\
\hline $30-06-2020$ & 108 & -1.75 & Down Swing \\
\hline
\end{tabular}


Model for Identifying Volatility in Equity Market with the Help of NSE Listed Stocks with Special

Reference to Apollo Tyres, Asian Paints and Ashok Leyland during Post Covid-19 in India - A Case

\begin{tabular}{|c|c|c|c|}
\hline \multicolumn{4}{|c|}{ Study } \\
\hline $29-06-2020$ & 108.45 & 0.45 & Up Swing \\
\hline $26-06-2020$ & 111 & 2.55 & Up Swing \\
\hline $25-06-2020$ & 112.3 & 1.3 & Up Swing \\
\hline $24-06-2020$ & 108.75 & -3.55 & Down Swing \\
\hline $23-06-2020$ & 112.05 & 3.3 & Up Swing \\
\hline $22-06-2020$ & 111.9 & -0.15 & Down Swing \\
\hline $19-06-2020$ & 109 & -2.9 & Down Swing \\
\hline $18-06-2020$ & 106.05 & -2.95 & Down Swing \\
\hline $17-06-2020$ & 105.35 & -0.7 & Down Swing \\
\hline $16-06-2020$ & 105.8 & 0.45 & Up Swing \\
\hline $15-06-2020$ & 106.9 & 1.1 & Up Swing \\
\hline $12 / 6 / 2020$ & 106.65 & -0.25 & Down Swing \\
\hline $11 / 6 / 2020$ & 105 & -1.65 & Down Swing \\
\hline $10 / 6 / 2020$ & 105.65 & 0.65 & Up Swing \\
\hline $9 / 6 / 2020$ & 104.75 & -0.9 & Down Swing \\
\hline $8 / 6 / 2020$ & 106.35 & 1.6 & Up Swing \\
\hline $5 / 6 / 2020$ & 104.7 & -1.65 & Down Swing \\
\hline $4 / 6 / 2020$ & 102.7 & -2 & Down Swing \\
\hline $3 / 6 / 2020$ & 107.15 & 4.45 & Up Swing \\
\hline $2 / 6 / 2020$ & 103.95 & -3.2 & Down Swing \\
\hline $1 / 6 / 2020$ & 104.75 & 0.8 & Up Swing \\
\hline $29-05-2020$ & 97.1 & -7.65 & Down Swing \\
\hline $28-05-2020$ & 98.05 & 0.95 & Up Swing \\
\hline $27-05-2020$ & 94.85 & -3.2 & Down Swing \\
\hline $26-05-2020$ & 92.35 & -2.5 & Down Swing \\
\hline $22-05-2020$ & 90.35 & -2 & Down Swing \\
\hline $21-05-2020$ & 91.85 & 1.5 & Up Swing \\
\hline $20-05-2020$ & 90.35 & -1.5 & Down Swing \\
\hline $19-05-2020$ & 91.8 & 1.45 & Up Swing \\
\hline $18-05-2020$ & 91.65 & -0.15 & Down Swing \\
\hline $15-05-2020$ & 91.7 & 0.05 & Up Swing \\
\hline $14-05-2020$ & 92.6 & 0.9 & Up Swing \\
\hline $13-05-2020$ & 94.4 & 1.8 & Up Swing \\
\hline $12 / 5 / 2020$ & 90.95 & -3.45 & Down Swing \\
\hline $11 / 5 / 2020$ & 93.1 & 2.15 & Up Swing \\
\hline $8 / 5 / 2020$ & 90.1 & -3 & Up Swing \\
\hline $7 / 5 / 2020$ & 89.65 & -0.45 & Down Swing \\
\hline $6 / 5 / 2020$ & 87.1 & -2.55 & Down Swing \\
\hline $5 / 5 / 2020$ & 85.7 & -1.4 & Down Swing \\
\hline $4 / 5 / 2020$ & 88.75 & 3.05 & Up Swing \\
\hline $30-04-2020$ & 96.3 & 7.55 & Up Swing \\
\hline $29-04-2020$ & 95.65 & -0.65 & Down Swing \\
\hline $28-04-2020$ & 89.5 & -6.15 & Down Swing \\
\hline $27-04-2020$ & 89.7 & 0.2 & Up Swing \\
\hline $24-04-2020$ & 89.95 & 0.25 & Up Swing \\
\hline $23-04-2020$ & 93.25 & 3.3 & Up Swing \\
\hline $22-04-2020$ & 94.35 & 1.1 & Up Swing \\
\hline $21-04-2020$ & 91.95 & -2.4 & Down Swing \\
\hline $20-04-2020$ & 97.35 & 5.4 & Up Swing \\
\hline $17-04-2020$ & 98.2 & 0.85 & Up Swing \\
\hline $16-04-2020$ & 94 & -4.2 & Down Swing \\
\hline $15-04-2020$ & 88.15 & -5.85 & Down Swing \\
\hline $13-04-2020$ & 85.8 & -2.35 & Down Swing \\
\hline $9 / 4 / 2020$ & 89.8 & 4 & Up Swing \\
\hline $8 / 4 / 2020$ & 83.4 & -6.4 & Down Swing \\
\hline $7 / 4 / 2020$ & 83.5 & 0.1 & Up Swing \\
\hline $3 / 4 / 2020$ & 78.45 & -5.05 & Down Swing \\
\hline
\end{tabular}




\begin{tabular}{|l|l|l|l|}
\hline $1 / 4 / 2020$ & 77.9 & -0.55 & Down Swing \\
\hline $31-03-2020$ & 79.4 & 1.5 & Up Swing \\
\hline $30-03-2020$ & 81.6 & 2.2 & Up Swing \\
\hline $27-03-2020$ & 82.6 & 1 & Up Swing \\
\hline $26-03-2020$ & 82.4 & -0.2 & Down Swing \\
\hline $25-03-2020$ & 80.85 & -1.55 & Down Swing \\
\hline $24-03-2020$ & 78.55 & -2.3 & Down Swing \\
\hline $23-03-2020$ & 76.45 & -2.1 & Down Swing \\
\hline $20-03-2020$ & 88.85 & 12.4 & Up Swing \\
\hline $19-03-2020$ & 86.2 & -2.65 & Down Swing \\
\hline $18-03-2020$ & 89.4 & 3.2 & Up Swing \\
\hline $17-03-2020$ & 93.6 & 4.2 & Up Swing \\
\hline $16-03-2020$ & 97.6 & 4 & Up Swing \\
\hline $13-03-2020$ & 104.15 & 6.55 & Up Swing \\
\hline $12 / 3 / 2020$ & 100.15 & -4 & Down Swing \\
\hline $11 / 3 / 2020$ & 115 & 14.85 & Up Swing \\
\hline $9 / 3 / 2020$ & 126.75 & 11.75 & Up Swing \\
\hline $6 / 3 / 2020$ & 135.3 & 8.55 & Up Swing \\
\hline $5 / 3 / 2020$ & 139.25 & 3.95 & Up Swing \\
\hline $4 / 3 / 2020$ & 139.95 & 0.7 & Up Swing \\
\hline $3 / 3 / 2020$ & 141.75 & 1.8 & Up Swing \\
\hline $2 / 3 / 2020$ & 136.3 & -5.45 & Down Swing \\
\hline
\end{tabular}

\begin{tabular}{|l|l|l|}
\hline \multicolumn{1}{|c|}{ Month } & \multicolumn{1}{|c|}{ Num of Up Swing } & \multicolumn{1}{c|}{ Num of Down Swing } \\
\hline March & $14 \quad$ & 7 \\
\hline April & 9 & 9 \\
\hline May & 9 & 10 \\
\hline June & 10 & 12 \\
\hline July & 14 & 9 \\
\hline August & 11 & 10 \\
\hline September & 4 & 5 \\
\hline TOTAL & 71 & 62 \\
\hline
\end{tabular}

\subsection{Financial Result of Apollo Tyres Limited}

Table 2 Financial Result of Apollo Tyres Limited

\begin{tabular}{|l|l|l|l|l|}
\hline Financial Year & $\begin{array}{c}\text { Earnings Per } \\
\text { Share(Rs in Cr) }\end{array}$ & $\begin{array}{c}\text { Book value per } \\
\text { share(Rs in Cr) }\end{array}$ & $\begin{array}{c}\text { Return on } \\
\text { capital employed } \\
\text { (\%) }\end{array}$ & $\begin{array}{c}\text { Cash flow from } \\
\text { operating } \\
\text { activities(Cr) }\end{array}$ \\
\hline 2016 & 22.06 & 129.69 & 13.68 & 2122 \\
\hline 2017 & 21.59 & 143.22 & 10.13 & 902 \\
\hline 2018 & 13.43 & 170.91 & 4.76 & 1719 \\
\hline 2019 & 11.88 & 175.51 & 8.01 & 1071 \\
\hline
\end{tabular}

\section{Interpretation}

On analysing the financial results, EPS and ROCE are decreasing year by year which means the company not able to produce the return from the capital. On the other hand BVP and CFO is gradually increasing it means that company increase the cash on day to day business activities. Upswing is more when compared to downswing it means the stock is less risk to buy.Cash market it is safe to buy the stock, and in Derivative market also it is safe and worth to buy. 
Model for Identifying Volatility in Equity Market with the Help of NSE Listed Stocks with Special

Reference to Apollo Tyres, Asian Paints and Ashok Leyland during Post Covid-19 in India - A Case Study

\section{ASIAN PAINTS}

\subsection{Asian Paints Limited}

Is an Indian multinational paint company headquartered in Mumbai, Maharashtra. The company is engaged in the business of manufacturing, selling and distribution of paints, coatings, products related to home decor, bath fittings and providing of related services. Asian Paints is India's largest and Asia's third largest paints corporation. As of 2015, it has the largest market share with $54.1 \%$ in the Indian paint industry. Asian Paints is the holding company of Berger International.

\subsection{Closing Price on Cash Market}

Table 3 Closing Price on Cash Market

\begin{tabular}{|c|c|c|c|}
\hline Date & Closing Price & Change in Closing price & Swings \\
\hline 14-09-2020 & 2031.55 & & \\
\hline $11 / 9 / 2020$ & 2035.2 & 3.65 & Up Swing \\
\hline $10 / 9 / 2020$ & 2062.9 & 27.7 & Up Swing \\
\hline 9/9/2020 & 1978.8 & -84.1 & Down Swing \\
\hline $8 / 9 / 2020$ & 1958.15 & -20.65 & Down Swing \\
\hline $7 / 9 / 2020$ & 1981.95 & 23.8 & Up Swing \\
\hline $4 / 9 / 2020$ & 1956 & -25.95 & Down Swing \\
\hline $3 / 9 / 2020$ & 1978.45 & 22.45 & Up Swing \\
\hline $2 / 9 / 2020$ & 1946 & -32.45 & Down Swing \\
\hline $1 / 9 / 2020$ & 1978.2 & 32.2 & Up Swing \\
\hline $31-08-2020$ & 1903.4 & -74.8 & Down Swing \\
\hline $28-08-2020$ & 1957.7 & 54.3 & Up Swing \\
\hline $27-08-2020$ & 1982 & 24.3 & Up Swing \\
\hline $26-08-2020$ & 1971.05 & -10.95 & Down Swing \\
\hline $25-08-2020$ & 2001 & 29.95 & Up Swing \\
\hline $24-08-2020$ & 1963.25 & -37.75 & Down Swing \\
\hline $21-08-2020$ & 1958.35 & -4.9 & Down Swing \\
\hline $20-08-2020$ & 1875.25 & -83.1 & Down Swing \\
\hline 19-08-2020 & 1885.7 & 10.45 & Up Swing \\
\hline $18-08-2020$ & 1873.6 & -12.1 & Down Swing \\
\hline $17-08-2020$ & 1835.85 & -37.75 & Down Swing \\
\hline $14-08-2020$ & 1801.6 & -34.25 & Down Swing \\
\hline $13-08-2020$ & 1802.15 & 0.55 & Up Swing \\
\hline $12 / 8 / 2020$ & 1796.75 & -5.4 & Down Swing \\
\hline $11 / 8 / 2020$ & 1789.55 & -7.2 & Down Swing \\
\hline $10 / 8 / 2020$ & 1782.45 & -7.1 & Down Swing \\
\hline $7 / 8 / 2020$ & 1805.05 & 22.6 & Up Swing \\
\hline $6 / 8 / 2020$ & 1728.25 & -76.8 & Down Swing \\
\hline $5 / 8 / 2020$ & 1717.15 & -11.1 & Down Swing \\
\hline $4 / 8 / 2020$ & 1714.3 & -2.85 & Down Swing \\
\hline $3 / 8 / 2020$ & 1704.95 & -9.35 & Down Swing \\
\hline $31-07-2020$ & 1716.65 & 11.7 & Up Swing \\
\hline $30-07-2020$ & 1743.1 & 26.45 & Up Swing \\
\hline $29-07-2020$ & 1737.45 & -5.65 & Down Swing \\
\hline $28-07-2020$ & 1759.6 & 22.15 & Up Swing \\
\hline $27-07-2020$ & 1777.75 & 18.15 & Up Swing \\
\hline $24-07-2020$ & 1710.95 & -66.8 & Down Swing \\
\hline $23-07-2020$ & 1727.4 & 16.45 & Up Swing \\
\hline $22-07-2020$ & 1697.45 & -29.95 & Down Swing \\
\hline $21-07-2020$ & 1723.6 & 26.15 & Up Swing \\
\hline $20-07-2020$ & 1751.95 & 28.35 & Up Swing \\
\hline $17-07-2020$ & 1717.95 & -34 & Down Swing \\
\hline $16-07-2020$ & 1692.65 & -25.3 & Down Swing \\
\hline $15-07-2020$ & 1671.3 & -21.35 & Down Swing \\
\hline
\end{tabular}




\begin{tabular}{|c|c|c|c|}
\hline $14-07-2020$ & 1688.45 & 17.15 & Up Swing \\
\hline $13-07-2020$ & 1705.65 & 17.2 & Up Swing \\
\hline $10 / 7 / 2020$ & 1703.05 & -2.6 & Down Swing \\
\hline $9 / 7 / 2020$ & 1716.8 & 13.75 & Up Swing \\
\hline $8 / 7 / 2020$ & 1686.6 & -30.2 & Down Swing \\
\hline $7 / 7 / 2020$ & 1745.45 & 58.85 & Up Swing \\
\hline $6 / 7 / 2020$ & 1708.35 & -37.1 & Down Swing \\
\hline $3 / 7 / 2020$ & 1696.1 & -12.25 & Down Swing \\
\hline $2 / 7 / 2020$ & 1686.7 & -9.4 & Down Swing \\
\hline $1 / 7 / 2020$ & 1688.1 & 1.4 & Up Swing \\
\hline $30-06-2020$ & 1687.35 & -0.75 & Down Swing \\
\hline $29-06-2020$ & 1691.95 & 4.6 & Up Swing \\
\hline $26-06-2020$ & 1687.35 & -4.6 & Down Swing \\
\hline $25-06-2020$ & 1688.85 & 1.5 & Up Swing \\
\hline $24-06-2020$ & 1746.4 & 57.55 & Up Swing \\
\hline $23-06-2020$ & 1683.65 & -62.75 & Down Swing \\
\hline $22-06-2020$ & 1653.45 & -30.2 & Down Swing \\
\hline 19-06-2020 & 1622.25 & -31.2 & Down Swing \\
\hline $18-06-2020$ & 1598.9 & -23.35 & Down Swing \\
\hline $17-06-2020$ & 1596.1 & -2.8 & Down Swing \\
\hline $16-06-2020$ & 1621.05 & 24.95 & Up Swing \\
\hline $15-06-2020$ & 1618.05 & -3 & Down Swing \\
\hline $12 / 6 / 2020$ & 1637.95 & 19.9 & Up Swing \\
\hline $11 / 6 / 2020$ & 1609.55 & -28.4 & Down Swing \\
\hline $10 / 6 / 2020$ & 1636.2 & 26.65 & Up Swing \\
\hline $9 / 6 / 2020$ & 1641.55 & 5.35 & Up Swing \\
\hline $8 / 6 / 2020$ & 1634.45 & -7.1 & Down Swing \\
\hline $5 / 6 / 2020$ & 1638.4 & 3.95 & Up Swing \\
\hline $4 / 6 / 2020$ & 1633 & -5.4 & Down Swing \\
\hline $3 / 6 / 2020$ & 1716.3 & 83.3 & Up Swing \\
\hline $2 / 6 / 2020$ & 1709.85 & -6.45 & Down Swing \\
\hline $1 / 6 / 2020$ & 1693.5 & -16.35 & Down Swing \\
\hline $29-05-2020$ & 1684.4 & -9.1 & Down Swing \\
\hline $28-05-2020$ & 1643.75 & -40.65 & Down Swing \\
\hline $27-05-2020$ & 1619.75 & -24 & Down Swing \\
\hline $26-05-2020$ & 1630.5 & 10.75 & Up Swing \\
\hline $22-05-2020$ & 1617.85 & -12.65 & Down Swing \\
\hline $21-05-2020$ & 1575 & -42.85 & Down Swing \\
\hline $20-05-2020$ & 1500.45 & -74.55 & Down Swing \\
\hline $19-05-2020$ & 1505.75 & 5.3 & Up Swing \\
\hline $18-05-2020$ & 1501.15 & -4.6 & Down Swing \\
\hline $15-05-2020$ & 1553.75 & 52.6 & Up Swing \\
\hline $14-05-2020$ & 1522.15 & -31.6 & Down Swing \\
\hline $13-05-2020$ & 1562.9 & 40.75 & Up Swing \\
\hline $12 / 5 / 2020$ & 1523.2 & -39.7 & Down Swing \\
\hline $11 / 5 / 2020$ & 1569.25 & 46.05 & Up Swing \\
\hline $8 / 5 / 2020$ & 1577.9 & 8.65 & Up Swing \\
\hline $7 / 5 / 2020$ & 1593.95 & 16.05 & Up Swing \\
\hline $6 / 5 / 2020$ & 1610.6 & 16.65 & Up Swing \\
\hline $5 / 5 / 2020$ & 1618.55 & 7.95 & Up Swing \\
\hline $4 / 5 / 2020$ & 1676.2 & 57.65 & Up Swing \\
\hline $30-04-2020$ & 1760.05 & 83.85 & Up Swing \\
\hline $29-04-2020$ & 1768 & 7.95 & Up Swing \\
\hline $28-04-2020$ & 1824.2 & 56.2 & Up Swing \\
\hline $27-04-2020$ & 1852.3 & 28.1 & Up Swing \\
\hline $24-04-2020$ & 1827.4 & -24.9 & Down Swing \\
\hline $23-04-2020$ & 1820.4 & $\begin{array}{l}-7 \\
\end{array}$ & Down Swing \\
\hline $22-04-2020$ & 1812.65 & -7.75 & Down Swing \\
\hline $21-04-2020$ & 1725.6 & -87.05 & Down Swing \\
\hline $20-04-2020$ & 1731.65 & 6.05 & Up Swing \\
\hline $17-04-2020$ & 1754.6 & 22.95 & Up Swing \\
\hline
\end{tabular}


Model for Identifying Volatility in Equity Market with the Help of NSE Listed Stocks with Special

Reference to Apollo Tyres, Asian Paints and Ashok Leyland during Post Covid-19 in India - A Case

\begin{tabular}{|c|c|c|c|c|}
\hline \multicolumn{5}{|c|}{ Study } \\
\hline $16-04-2020$ & 1742.35 & \multicolumn{2}{|l|}{-12.25} & Down Swing \\
\hline $15-04-2020$ & 1718.4 & \multicolumn{2}{|l|}{-23.95} & Down Swing \\
\hline 13-04-2020 & 1679.35 & \multicolumn{2}{|l|}{-39.05} & Down Swing \\
\hline $9 / 4 / 2020$ & 1652.45 & \multicolumn{2}{|l|}{-26.9} & Down Swing \\
\hline $8 / 4 / 2020$ & 1609.6 & \multicolumn{2}{|l|}{-42.85} & Down Swing \\
\hline $7 / 4 / 2020$ & 1624.05 & \multicolumn{2}{|l|}{14.45} & Up Swing \\
\hline $3 / 4 / 2020$ & 1521.3 & \multicolumn{2}{|l|}{-102.75} & Down Swing \\
\hline $1 / 4 / 2020$ & 1603.9 & \multicolumn{2}{|l|}{82.6} & Up Swing \\
\hline $31-03-2020$ & 1666.7 & \multicolumn{2}{|l|}{62.8} & Up Swing \\
\hline $30-03-2020$ & 1597.15 & \multicolumn{2}{|l|}{-69.55} & Down Swing \\
\hline $27-03-2020$ & 1606.2 & \multicolumn{2}{|l|}{9.05} & Up Swing \\
\hline $26-03-2020$ & 1629.7 & \multicolumn{2}{|l|}{23.5} & Up Swing \\
\hline $25-03-2020$ & 1594.05 & \multicolumn{2}{|l|}{-35.65} & Down Swing \\
\hline $24-03-2020$ & 1525.25 & -68.8 & & Down Swing \\
\hline $23-03-2020$ & 1498.75 & -26.5 & & Down Swing \\
\hline $20-03-2020$ & 1745.85 & 247.1 & & Up Swing \\
\hline 19-03-2020 & 1603 & -142.85 & & Down Swing \\
\hline $18-03-2020$ & 1616.65 & 13.65 & & Up Swing \\
\hline $17-03-2020$ & 1734.2 & 117.55 & & Up Swing \\
\hline $16-03-2020$ & 1682.95 & -51.25 & & Down Swing \\
\hline $13-03-2020$ & 1797.6 & 114.65 & & Up Swing \\
\hline $12 / 3 / 2020$ & 1842.85 & 45.25 & & Up Swing \\
\hline $11 / 3 / 2020$ & 1882.65 & 39.8 & & Up Swing \\
\hline $9 / 3 / 2020$ & 1869.45 & -13.2 & & Down Swing \\
\hline $6 / 3 / 2020$ & 1878.5 & 9.05 & & Up Swing \\
\hline $5 / 3 / 2020$ & 1875.45 & -3.05 & & Down Swing \\
\hline $4 / 3 / 2020$ & 1849.85 & -25.6 & & Down Swing \\
\hline $3 / 3 / 2020$ & 1801.65 & -48.2 & & Down Swing \\
\hline $2 / 3 / 2020$ & 1788.55 & -13.1 & & Down Swing \\
\hline & & n of Up Swing & & Num of Down Swing \\
\hline March & 10 & & 11 & \\
\hline April & 8 & & 10 & \\
\hline May & 10 & & 9 & \\
\hline June & 9 & & 13 & \\
\hline July & 12 & & 11 & \\
\hline August & 6 & & 15 & \\
\hline September & 5 & & 4 & \\
\hline TOTAL & 60 & & 73 & \\
\hline
\end{tabular}

\subsection{Financial Results of Asian Paints Limited}

Table 4

\begin{tabular}{|c|c|c|c|c|}
\hline Financial Year & $\begin{array}{c}\text { Earnings Per } \\
\text { Share(Rs in Cr) }\end{array}$ & $\begin{array}{c}\text { Book value per } \\
\text { share(Rs in Cr) }\end{array}$ & $\begin{array}{c}\text { Return on capital } \\
\text { employed (\%) }\end{array}$ & $\begin{array}{c}\text { Cash flow from } \\
\text { operating } \\
\text { activities(Cr) }\end{array}$ \\
\hline 2016 & 18.19 & 72.02 & 23.54 & 2242 \\
\hline 2017 & 20.22 & 83.19 & 34.13 & 1527 \\
\hline 2018 & 21.26 & 91.10 & 32.76 & 2113 \\
\hline 2019 & 22.48 & 102.50 & 30.42 & 2469 \\
\hline 2020 & 28.20 & 109.82 & 31.30 & 2631 \\
\hline
\end{tabular}

\section{Interpretation}

On analyzing the financial results, EPS, BVP, ROCE and CFO all are increasing for the past five years but here down swing is more when compared to upswing it shows that the stock is 
safe for long term investors and it is risk for short term investors. In Cash market it is risky to buy the stock.

\section{ASHOK LEYLAND}

\subsection{Ashok Leyland}

Is an Indian automobile company headquartered in Chennai, India. It is owned by the Hinduja Group. Founded in 1948, it is the second largest commercial vehicle manufacturer in India, fourth largest manufacturer of buses in the world and 10th largest manufacturer of trucks globally. Operating nine plants, Ashok Leyland also makes spare parts and engines for industrial and marine applications. It is the second largest commercial vehicle company in India in the medium and heavy commercial vehicle (M\&HCV) segment, with a market share of $74.65 \%$

\subsection{Closing Price on Cash Market}

Table 5 Closing Price on Cash Market

\begin{tabular}{|c|c|c|c|}
\hline Date & Closing Price & Change in Closing Price & Swing \\
\hline $14-09-2020$ & 74.8 & & \\
\hline $11 / 9 / 2020$ & 68.15 & -6.65 & Down Swing \\
\hline $10 / 9 / 2020$ & 67.75 & -0.4 & Down Swing \\
\hline $9 / 9 / 2020$ & 66.95 & -0.8 & Down Swing \\
\hline $8 / 9 / 2020$ & 67.55 & 0.6 & Up Swing \\
\hline $7 / 9 / 2020$ & 69.55 & 2 & Up Swing \\
\hline $4 / 9 / 2020$ & 69.8 & 0.25 & Up Swing \\
\hline $3 / 9 / 2020$ & 69.5 & -0.3 & Down Swing \\
\hline $2 / 9 / 2020$ & 68.45 & -1.05 & Down Swing \\
\hline $1 / 9 / 2020$ & 68.8 & 0.35 & Up Swing \\
\hline $31-08-2020$ & 67.65 & -1.15 & Down Swing \\
\hline $28-08-2020$ & 70.5 & 2.85 & Up Swing \\
\hline $27-08-2020$ & 72.5 & 2 & Up Swing \\
\hline $26-08-2020$ & 71.05 & -1.45 & Down Swing \\
\hline $25-08-2020$ & 69.95 & -1.1 & Down Swing \\
\hline $24-08-2020$ & 68.4 & -1.55 & Down Swing \\
\hline $21-08-2020$ & 69.35 & 0.95 & Up Swing \\
\hline $20-08-2020$ & 71.3 & 1.95 & Up Swing \\
\hline $19-08-2020$ & 69.25 & -2.05 & Down Swing \\
\hline $18-08-2020$ & 68.5 & -0.75 & Down Swing \\
\hline $17-08-2020$ & 62.7 & -5.8 & Down Swing \\
\hline $14-08-2020$ & 61.6 & -1.1 & Down Swing \\
\hline $13-08-2020$ & 61.1 & -0.5 & Down Swing \\
\hline $12 / 8 / 2020$ & 53.95 & -7.15 & Down Swing \\
\hline $11 / 8 / 2020$ & 52.2 & -1.75 & Down Swing \\
\hline wae $10 / 8 / 2020$ & 51.4 & -0.8 & Down Swing \\
\hline $7 / 8 / 2020$ & 49.95 & -1.45 & Down Swing \\
\hline $6 / 8 / 2020$ & 49.05 & -0.9 & Down Swing \\
\hline $5 / 8 / 2020$ & 49 & -0.05 & Down Swing \\
\hline $4 / 8 / 2020$ & 49.6 & 0.6 & Up Swing \\
\hline $3 / 8 / 2020$ & 49.3 & -0.3 & Down Swing \\
\hline $31-07-2020$ & 48.65 & -0.65 & Down Swing \\
\hline $30-07-2020$ & 49.15 & 0.5 & Up Swing \\
\hline $29-07-2020$ & 51.2 & 2.05 & Up Swing \\
\hline $28-07-2020$ & 51.15 & -0.05 & Down Swing \\
\hline $27-07-2020$ & 49.35 & -1.8 & Down Swing \\
\hline $24-07-2020$ & 50.4 & 1.05 & Up Swing \\
\hline
\end{tabular}


Model for Identifying Volatility in Equity Market with the Help of NSE Listed Stocks with Special

Reference to Apollo Tyres, Asian Paints and Ashok Leyland during Post Covid-19 in India - A Case Study

\begin{tabular}{|c|c|c|c|}
\hline $23-07-2020$ & 52 & 1.6 & Up Swing \\
\hline $22-07-2020$ & 51.65 & -0.35 & Down Swing \\
\hline $21-07-2020$ & 52.45 & 0.8 & Up Swing \\
\hline $20-07-2020$ & 51.55 & -0.9 & Down Swing \\
\hline $17-07-2020$ & 51.75 & 0.2 & Up Swing \\
\hline $16-07-2020$ & 50.2 & -1.55 & Down Swing \\
\hline $15-07-2020$ & 49.3 & -0.9 & Down Swing \\
\hline $14-07-2020$ & 50.4 & 1.1 & Up Swing \\
\hline $13-07-2020$ & 50.8 & 0.4 & Up Swing \\
\hline $10 / 7 / 2020$ & 51.15 & 0.35 & Up Swing \\
\hline 9/7/2020 & 52.15 & 1 & Up Swing \\
\hline $8 / 7 / 2020$ & 51.95 & -0.2 & Down Swing \\
\hline $7 / 7 / 2020$ & 51.9 & -0.05 & Down Swing \\
\hline $6 / 7 / 2020$ & 51.75 & -0.15 & Down Swing \\
\hline $3 / 7 / 2020$ & 49.1 & -2.65 & Down Swing \\
\hline $2 / 7 / 2020$ & 49.1 & 0 & Up Swing \\
\hline $1 / 7 / 2020$ & 48.2 & -0.9 & Down Swing \\
\hline $30-06-2020$ & 46.95 & -1.25 & Down Swing \\
\hline $29-06-2020$ & 48.6 & 1.65 & Up Swing \\
\hline $26-06-2020$ & 52.45 & 3.85 & Up Swing \\
\hline $25-06-2020$ & 53.45 & 1 & Up Swing \\
\hline $24-06-2020$ & 54.15 & 0.7 & Up Swing \\
\hline $23-06-2020$ & 56.25 & 2.1 & Up Swing \\
\hline $22-06-2020$ & 54.6 & -1.65 & Down Swing \\
\hline $19-06-2020$ & 54.35 & -0.25 & Down Swing \\
\hline $18-06-2020$ & 51.15 & -3.2 & Down Swing \\
\hline $17-06-2020$ & 50.5 & -0.65 & Down Swing \\
\hline $16-06-2020$ & 50.85 & 0.35 & Up Swing \\
\hline $15-06-2020$ & 51.75 & 0.9 & Up Swing \\
\hline $12 / 6 / 2020$ & 52.35 & 0.6 & Up Swing \\
\hline $11 / 6 / 2020$ & 51.1 & -1.25 & Down Swing \\
\hline $10 / 6 / 2020$ & 52.7 & 1.6 & Up Swing \\
\hline $9 / 6 / 2020$ & 50.75 & -1.95 & Down Swing \\
\hline $8 / 6 / 2020$ & 53.05 & 2.3 & Up Swing \\
\hline $5 / 6 / 2020$ & 48.35 & -4.7 & Down Swing \\
\hline $4 / 6 / 2020$ & 45.75 & -2.6 & Down Swing \\
\hline $3 / 6 / 2020$ & 47.15 & 1.4 & Up Swing \\
\hline $2 / 6 / 2020$ & 47.7 & 0.55 & Up Swing \\
\hline $1 / 6 / 2020$ & 46.4 & -1.3 & Down Swing \\
\hline $29-05-2020$ & 42.45 & -3.95 & Down Swing \\
\hline $28-05-2020$ & 44.4 & 1.95 & Up Swing \\
\hline $27-05-2020$ & 43.2 & -1.2 & Down Swing \\
\hline $26-05-2020$ & 43.55 & 0.35 & Up Swing \\
\hline $22-05-2020$ & 43.6 & 0.05 & Up Swing \\
\hline $21-05-2020$ & 46.1 & 2.5 & Up Swing \\
\hline $20-05-2020$ & 43.35 & -2.75 & Down Swing \\
\hline $19-05-2020$ & 43.05 & -0.3 & Down Swing \\
\hline $18-05-2020$ & 43.85 & 0.8 & Up Swing \\
\hline $15-05-2020$ & 47.15 & 3.3 & Up Swing \\
\hline $14-05-2020$ & 49.3 & 2.15 & Up Swing \\
\hline $13-05-2020$ & 48.75 & -0.55 & Down Swing \\
\hline $12 / 5 / 2020$ & 48.6 & -0.15 & Down Swing \\
\hline $11 / 5 / 2020$ & 48.75 & 0.15 & Up Swing \\
\hline $8 / 5 / 2020$ & 47.05 & -1.7 & Down Swing \\
\hline $7 / 5 / 2020$ & 46.1 & -0.95 & Down Swing \\
\hline $6 / 5 / 2020$ & 47.6 & 1.5 & Up Swing \\
\hline $5 / 5 / 2020$ & 48.35 & 0.75 & Up Swing \\
\hline
\end{tabular}




\begin{tabular}{|c|c|c|c|}
\hline $4 / 5 / 2020$ & 49.3 & 0.95 & Up Swing \\
\hline $30-04-2020$ & 52.35 & 3.05 & Up Swing \\
\hline 29-04-2020 & 47.95 & -4.4 & Down Swing \\
\hline $28-04-2020$ & 44.55 & -3.4 & Down Swing \\
\hline $27-04-2020$ & 44.3 & -0.25 & Down Swing \\
\hline 24-04-2020 & 44.95 & 0.65 & Up Swing \\
\hline $23-04-2020$ & 46.7 & 1.75 & Up Swing \\
\hline $22-04-2020$ & 44.95 & -1.75 & Down Swing \\
\hline 21-04-2020 & 44.1 & -0.85 & Down Swing \\
\hline $20-04-2020$ & 47.9 & 3.8 & Up Swing \\
\hline $17-04-2020$ & 48.9 & 1 & Up Swing \\
\hline $16-04-2020$ & 46.95 & -1.95 & Down Swing \\
\hline $15-04-2020$ & 46.7 & -0.25 & Down Swing \\
\hline 13-04-2020 & 46.05 & -0.65 & Down Swing \\
\hline $9 / 4 / 2020$ & 45.65 & -0.4 & Down Swing \\
\hline $8 / 4 / 2020$ & 43.85 & -1.8 & Down Swing \\
\hline $7 / 4 / 2020$ & 39.85 & -4 & Down Swing \\
\hline $3 / 4 / 2020$ & 38.15 & -1.7 & Down Swing \\
\hline $1 / 4 / 2020$ & 41.1 & 2.95 & Up Swing \\
\hline $31-03-2020$ & 43.05 & 1.95 & Up Swing \\
\hline $30-03-2020$ & 42.35 & -0.7 & Down Swing \\
\hline $27-03-2020$ & 43.3 & 0.95 & Up Swing \\
\hline $26-03-2020$ & 42.85 & -0.45 & Down Swing \\
\hline $25-03-2020$ & 34.45 & -8.4 & Down Swing \\
\hline $24-03-2020$ & 35.55 & 1.1 & Up Swing \\
\hline $23-03-2020$ & 36.05 & 0.5 & Up Swing \\
\hline $20-03-2020$ & 43.95 & 7.9 & Up Swing \\
\hline 19-03-2020 & 48.05 & 4.1 & Up Swing \\
\hline $18-03-2020$ & 64.25 & 16.2 & Up Swing \\
\hline $17-03-2020$ & 64.15 & -0.1 & Down Swing \\
\hline $16-03-2020$ & 63.35 & -0.8 & Down Swing \\
\hline $13-03-2020$ & 66.7 & 3.35 & Up Swing \\
\hline $12 / 3 / 2020$ & 63.3 & -3.4 & Down Swing \\
\hline $11 / 3 / 2020$ & 68.55 & 5.25 & Up Swing \\
\hline $9 / 3 / 2020$ & 68.85 & 0.3 & Up Swing \\
\hline $6 / 3 / 2020$ & 71.2 & 2.35 & Up Swing \\
\hline $5 / 3 / 2020$ & 71.8 & 0.6 & Up Swing \\
\hline $4 / 3 / 2020$ & 72.45 & 0.65 & Up Swing \\
\hline $3 / 3 / 2020$ & 75.45 & 3 & Up Swing \\
\hline $2 / 3 / 2020$ & 73.9 & -1.55 & Down Swing \\
\hline
\end{tabular}

\begin{tabular}{|l|l|l|}
\hline \multicolumn{1}{|c|}{ Month } & \multicolumn{1}{c|}{ Num of Up Swing } & Num of Down Swing \\
\hline March & 14 & 7 \\
\hline April & 6 & 12 \\
\hline May & 11 & 8 \\
\hline June & 12 & 10 \\
\hline July & 11 & 12 \\
\hline August & 5 & 16 \\
\hline September & 4 & 5 \\
\hline TOTAL & 63 & 70 \\
\hline
\end{tabular}


Model for Identifying Volatility in Equity Market with the Help of NSE Listed Stocks with Special Reference to Apollo Tyres, Asian Paints and Ashok Leyland during Post Covid-19 in India - A Case Study

\subsection{Financial Results of Ashok Leyland Limited}

Table 6 Financial Results of Ashok Leyland Limited

\begin{tabular}{|c|c|c|c|c|}
\hline Financial Year & $\begin{array}{c}\text { Earnings Per } \\
\text { Share(Rs in Cr) }\end{array}$ & $\begin{array}{c}\text { Book value per } \\
\text { share(Rs in Cr) }\end{array}$ & $\begin{array}{c}\text { Return on } \\
\text { capital employed } \\
\text { (\%) }\end{array}$ & $\begin{array}{c}\text { Cash flow from } \\
\text { operating } \\
\text { activities(Cr) }\end{array}$ \\
\hline 2016 & 2.40 & 19.96 & 4.88 & -1.274 \\
\hline 2017 & 5.51 & 24.53 & 17.52 & 270 \\
\hline 2018 & 6.02 & 28.17 & 19.67 & 1477 \\
\hline 2019 & 7.08 & 33.45 & 18.32 & -3745 \\
\hline 2020 & 1.15 & 30.30 & 11.10 & 383 \\
\hline
\end{tabular}

\section{Interpretation}

On analyzing the financial results, there is an erratic trend in EPS, BVPS, ROCE and CFO this indicates the company does not generating the profit from the capital. Down swing is more when compared to the upswing it means the stock is not safe and it is not fit to buy. In Cash market it is risky to buy the stock.

\section{CONCLUSION}

Equity Market is in fact a reflection of both Individual Companies' financial performance and Stock Market reaction to news and events. Therefore a stock price is the function of EPS, BVPS, ROCE, CFO and Trends in the Market. In this context, when in the case of Apollo Tyres Limited and Asian Paints Limited, It is found that both are Investors' friendly stocks in India. All the four above mentioned Financial Performance Indicators were in the positive trend and the price trend is also in favour of these two companies as it is witnessed from the reading more upswings than downswings but it is otherwise in the case of Ashok Leyland Limited on account of falling or fluctuating trend in all the four financial performance indicators namely EPS, BVPS, ROCE and CFO besides, the size swings in its stock price is found to be more down swings than upswings. Hence Ashok Leyland limited can not be resorted to buy but it can be put on hold.

\section{REFERENCES}

[1] Apollo Tyres-Annual Reports https://www.apollotyres.com/en-in/car-suv-van/tyre-finder/

[2] Ashokleyland-Annual Reports https://www.ashokleyland.com/en/web/ashokleyland/home

[3] Asian Paints-Annual Reports: https://www.asianpaints.com/annualreports

[4] Financial Ratios and definitions https : //www.investopedia.com/

[5] Stock Prices for Apollo Tyres, Ashok Leyland and Asian Paints https:

//www.nseindia.com/get - quotes/equity?symbol = AXISBANK https :

//www.nseindia.com/get - quotes/equity?symbol = BAJAJFINSV https :

$/ /$ www.nseindia.com/get - quotes/equity?symbol $=$ BAJAJ - AUTO

[6] Future Prices for past 90 days

https://www1.nseindia.com/products/content/derivatives/equities/historicalf o.htm

[7] Barua S K \&Varma J R (1991b), "Mastershares: A Bonanza for Large Investors", Vikalpa Vol. 16, No. 1 (Jan- Mar), p. 29-34. 3.

[8] Barua S K \&Varma J R (1991a), "Indian Convertible Bonds with Unspecified Terms: A Valuation Model", Working Paper No. 991, (Jul-Sep), Indian Institute of Management, Ahmedabad.

[9] www.moneycontrol.com 